\title{
Über den Einfluss der kolloidalen Lösung auf die Wasserab= sonderung in der isolierten Niere in normalen und pathologischen Zuständen.
}

(Studien über Flüssigkeitsaustausch. XXXX.)

\author{
Von
}

Koichi Aida.

(會田宏一)

(Aus der Medizinischen Klinik von Prof. Dr. T. Kato, Tohoku Reichsuniversität zu Sendai.)

Kolloidale Lösungen üben vorwiegend durch ihre physikalisch-chemische Einwirkung bedeutsame Einflüsse auf die Harnbildung. Nach

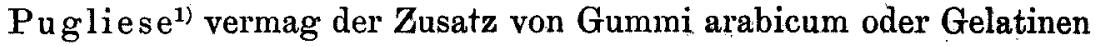
zu hypertonischer Kochsalzlösung diuretische Wirkung derselben zeitlich zu verzögern, und intravenöse Injektionen von reiner Gummilösung. und Gelatinlösung sollen stark antidiuretisch wirken, Knowlton ${ }^{2)}$ Turmaki u. Kurosawa ${ }^{3)}$, Hori'), Toyoshima, Yamakoshi') haben die Beobachtungen gemacht, dass an Kaninchen und Hunden durch Injektionen von physiologischer Kochsalzlösung oder Ringerlösung mit Gummi- oder Gelatinzusatz eine Verminderung der Harnmenge eintrat. Roger u. Garnièr haben konstatieit, dass die Diurese, welche durch Injektion von Lockscher Lösung bewirkt wird, durch Gummizusatz zu derselben erlischt. Cori ${ }^{8)}$ hat an Diabetiker den $\mathrm{Nach}$ weis erbracht, dass bei interavenöser Injektion von $7 \%$ iger Gummilösung gleichzeitig mit verminderter Zuckerausscheidung auch die Harnmenge beträchtlich abnahm.

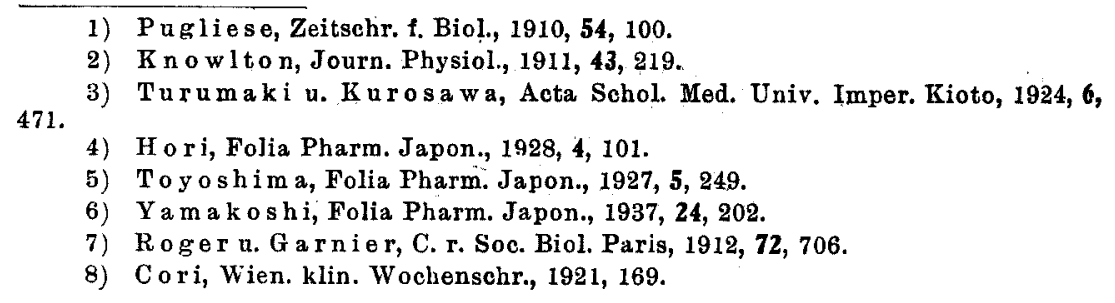


Nach alledern ist es klar, dass verchiedene kolloidale Substanzen die physikochemische Beschaffenheit des Blutes verändern und so auf den Wasseraustausch zwischen Blut und Geweben einen grossen Einfluss ausïben. Wenn daher kolloidalen Substanzen die antidiuretische Wirkung zukommt, ist es selbstverständlich, in erster Linie an die extrarenale Wirkung derselben zu denken. Indessen muss man aber auch annehmen, dass derarartige kolloidale Wirkung nicht allein extrarenal, sondern auch in Glomerulusschlingen und Tubuluslumen innerhalb der Niere zur Geltung kommt. Unter diesem Gesichtspunkt erschien mir sehr interessant, auf diese intrarenale Wirkung der kolloidalen Substanz zu fahnden.

In der Absicht, unter Ausschaltung extrarenaler Faktoren nur intrareanle Wirkung der kolloidalen Substanzen allein zu analysieren, wurde an isolierten überlebenden Krötennieren die Durchspülung mit kolloidallen Lösungen vorgenommen und darïber untersucht, ob und inwieweit diese Massnahme die Harnbildung beeinflussen könnte. Da bei der Krötenniere, wie allgemein wohl bekannt ist, der Glomerulusapparat von der Nierenarterie, die Harnkanälchen aber von der $\mathrm{V}$. renoportalis unabhängig voneinander mit Blut versorgt sind, ist es dafür sehr geeignet, die Funktionen beider Organe gesondert zu untersuchen.

In vorliegenden Versuchen wurden an der gesunden Niere sowie an den experimentell geschädigten pathologischen Nieren Veränderungen der Harnabsonderung, welche bei Durchspülung mit kolloidaler Lösung entweder von dem glomerulören oder von dem tubulären Kreislauf aus oder aber bei gleichzeitiger Durchspülung von den beiden Systemen aus auftreten, analysiert und zugleich auch die Verhältnisse der sich dabei abspielenden Nierenfunktion geprüft.

Versuchsmethode: Versuchsmethode war dieselbe, wie sie in der XII. Mitteilung.9) einschlägiger Studien angewandt worden war. Als Versuchstier wurden die Kröten, die sich unter Bedingungen des Sommerlebens befanden, benützt. Behufs Herbeiführung der Nierenschädigung kamen Uranylnitrat und Kantharidin zur Anwendung.

Es wurden jeder einzelnen Kröte von 0,1\% iger Kantharidinazetatlösung je 0,2 bzw. $0,3 \mathrm{ccm}$ pro $100 \mathrm{~g}$ Körpergewicht ca. $24 \mathrm{Std}$. vor Versuchsbeginn, oder von $2 \%$ iger Uranylnitratlösung je $0,2 \mathrm{ccm}$ bzw. $0,5 \mathrm{ccm}$ pro $100 \mathrm{~g}$ Körpergewicht ungefähr $48 \mathrm{Std}$. vor Versuchsbeginn in den Lymphsack des Hinterschenkels injiziert. Die Technik für die

9) Ya magu ch i, Tohoku Journ. Exp. Med., 1932, 18, 392.

10) Tada u. Saito, Tbid., 1930, 15, 91 . 
Herstellung des isolierten überlebenden Nierenpräparates sowie dessen Durchspülungsmethode sind in der IX. Mitteilung ${ }^{10)}$ einschlägiger Studien ausführlich beschrieben. Die Gummi-Ringerlösung wurde in der Weise bereitet, dass Gummi-Arabicum (Merck) in einer Konzentration von $1 \%$ in Ringerlösung gelöst, nach mehrmaligem Filtrieren mit Natriumkarbonat aufsgenaueste neutralisiert wurde.

Erst 10 Min., nachdem die Krötenniere mit Normal-Ringer etwa $30 \mathrm{Min}$. lang durchspült worden war, wurden die Zufuhrmenge und Ausfuhrmenge der Ringerlösung sowie die Intervalle des Austropfens des Uretertropfens bestimmt, und erst dann, als die angenäherte Stetigkeit der obigen Grössen sichergestellt worden war, wurde NormalRinger auf eine $1 \%$ ige Gummi-Ringerlösung umgeschaltet. Nach diesem Verfahren wurden die Zufuhrmenge der Durchspülungsflüssigkeit zu A. renalis und zu $\nabla$. renoportalis sowie die Ausfuhrmenge aus $V$. cava post. alle 5 Minuten bestimmt. Weiterhin wurden auch die Intervalle des aus der in die Ureteren eingeführten Kanüle austropfenden Uretertropfens, sog. Blasenharnes mit Sekundenmesser gemessen. Je nach der Länge dieses Intervalles wird das Verhalten der Harnabsonderung als Diurese oder Oligurie betrachtet. Der Druck der von der Nierenarterie aus hineinfliessenden Flüssigkeit wurde auf $24 \mathrm{~cm} \mathrm{H}_{2} \mathrm{O}$, der Druck der von der Nierenpfortader aus hineinfliessenden Flüssigkeit auf $12 \mathrm{~cm} \mathrm{H}_{2} \mathrm{O}$ festgesetzt.

I. Einfluss der Durchspülung mit $1 \%$ iger GummiRingerlösung auf die Harnabsonderung.

1. Versuch an der intakten Niere.

a) Zufuhr von der Nierenarterie aus.

Zur Vereinfachung der Erörterung über den Ablauf der Harnabsonderung in allen Versuchsanordnungen wird übersichtshalber im folgenden der erste Zeitraum, wo die Durchspülung mit Normal-Ringer allein ausgeführt wurde, als I. Stadium, derjenige Zeitraum, wo die Durchspülung mit einer 1\%igen Gummi-Ringerlösung ausgeführt wurde, als II. Stadium, und solcher Zeitraum, wo die Spülflüssigkeit wieder auf Normal-Ringer umgeschaltet wurde, als III. Stadium bezeichnet.

Es wurde an 5 Versuchen die Durchspülung mit $1 \%$ iger GummiRinger von der Nierenarterie aus vorgenommen; im II. Stadium verzögerte sich das Intervall des Austropfens des Uretertropfens sogleich ausnahmslos, nach 30 Min. währender Durchspülung trat erhebliche 
Oligurie ein. Ein Versuchsbeispiel dafür ist mit Fig. 1. illustriert.

Beim Eintritt in "III. Stadium, wo die Durchspülung wieder mit NormalRinger ausgeführt wurde, fing an das Intervall des Austropfens des Uretertropfens, sog. Blasenharnes sich wieder sogleich zu verkürzen, dies war ein Ausdruck wiederhergestellter Harnbildung.* Da aber bei isolierten überlebenden Präparaten, wie sie in vorliegenden Versuchen angewendet wurden, die Harnbildung sich mit dem Zeitverlauf mehr oder minder zu verzögern pflegt, blieb das Intervall des Austropfens des Uretertropfens im gewissermassen protrahierten $\mathrm{Zu}-$ stand beinahe konstant. Im II. Stadium nahm die $\mathrm{Zu}$ fuhrmenge zur Nierenarterie ab, wurde aber im III. Stadium bei Durchspülung mit Normal-Ringer wiederhergestellt. Es kann zwar nicht geleugnet werden, dass die Abnahme der Zufuhrmenge durch Gefässwirkung einen Faktor für die oben erwähnte Oligurie darstellt. Dennoch kann diese Abnahme durchaus nicht als die Hauptursache dafür angesehen werden deshalb, weil der Grad der Oligurie im Verhältnis zur Abnahme weitgehend grösser ist. Im späteren

* Bei Durchspülung mit Ringerlösung muss die Herabsetzung der Zellakti vität durch den mit dem Zeitablauf immer stärker sich geltend machenden $\mathrm{O}_{2}$-Mangel berücksichtigt werden.
Fig. 1. Gesunde Niere; Durchspülung mit $1 \%$ iger Gummi-Ringerlösung durch Nierenarterie.

Kröte, 各, Körpergewicht $165 \mathrm{~g}$.

Gummi-Ringer

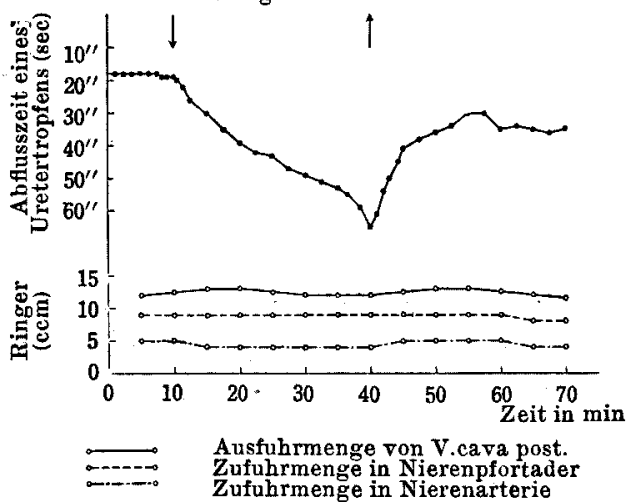

Fig. 2. Gesunde Niere; Durchspülung mit $1 \%$ iger Gummi-Ringerlösung durch Nierenpfortader.

Kröte, ชิ, Körpergewicht $110 \mathrm{~g}$.

Gummi-Ringer

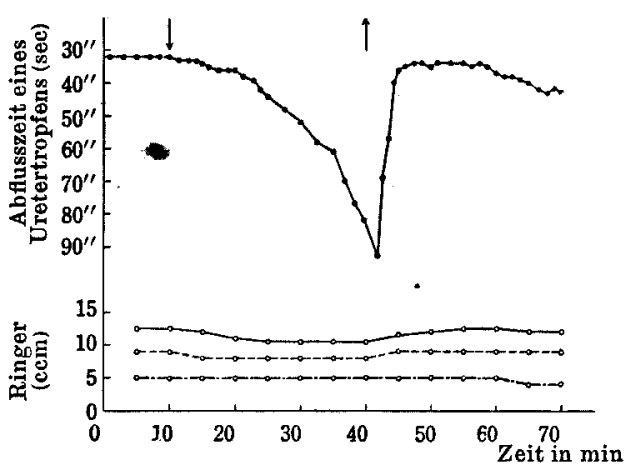

$\longrightarrow$ Ausfuhrmenge $\longrightarrow$ ron V. cara post.

Zufuhrmenge in Nierenarterie

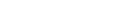


Stadium des Versuchs tendierte die Zufuhrmenge zur Nierenarterie mehr oder minder zur Abnahme.

b) Zufuhrvonder Nierenpfortaderaus.

Hierbei wurde die Durchspülung an 5 Versuchen ausgeführt. Ein Beispiel davon ist mit Fig. 2 illustriert.

Das Intervall des Austropfens des Uretertropfens, das im I. Stadium fast konstant verlief, zeigte sogleich nach Utbergang ins II. Stadium eklatante Verzögerung, welche nachher immer bemerkbarer wurde, nach Übergang in das III. Stadium aber wurde die lebhafte Harnbildung angetroffen. Hier ist zu bemerken, dass, trotzdem im II. Stadium die glomeruläre Zufuhrmenge unbeeinflusst blieb, die renoportale Zufuhrmenge abnahm, im III. Stadium jedoch auf den früheren Zustand zurückkam. Die Gefässwirkung liess sich hierbei nur an der Nierenpfortader in äusserst geringem Grad erkennen, während sie an der Nierenarterie gänzlich vermisst wurde.

c) Gleichzeitige Zufuhr van der Nierenarterie und von der Nierenpfortader aus.

Hierbei wurde die Durchspülung an 5 Versuchen durchgeführt.

Im II. Stadium trat die Oligurie in stärkerem Masse auf als bei allen vorerwähnten Massnahmen, gleichwohl wurde im III. Stadium die Harnbildung sehr prompt wiederhergestellt. Ein Beispiel dafür ist

Fig. 3. Gesunde Niere; Durchspülung mit $1 \%$ iger Gummi-Ringerlösung durch Nierenarterie und Nierenpfo mader. Kröte, 우, Körpergewicht $260 \mathrm{~g}$.

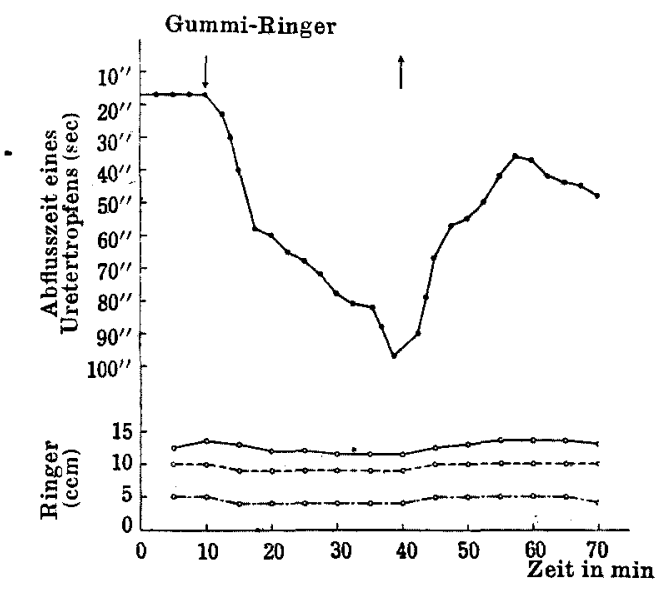

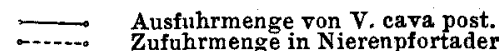
Zufuhrmenge in Nierenpfortad mit Fig. 3. illustriert. Die Abnahme der Zufulhrmenge im II. Stadium wurde sowohl an der Nierénárterie wie auch an der Nierenpfortader angetroffen, im .III. Stadium stellte sich die $\mathrm{Zu}$ fuhrmenge auf den früheren Zustand ein. Es kann aber gesagt werden, dass die hierbei vollzogene Gefässwirkung sich nur in gleichem Masse kundgab, wie die Summe der bei glomerulärer und tubulärer Durchspülung gesondert zustande gekommenen Gefässwirkung: Immerhin erwies sich der Grad der Oligurie im Ver- 
hältnis zur Abnahme der Zufuhrmenge bei weitem grösser, deshalb kann diese Oligurie entschieden durchaus nicht ausschliesslich oder vorwiegend auf die Gefässwirkung allein zurückgeführt werden.

\section{Versuch an der Kantharidinniere.}

a) Zufuhr von der Nierenarterie aus.

Hierbei wurde die Durchspülung an 5 Versuchen ausgeführt.

Der Einfluss der Gummi-Ringerlösung auf die Harnbildung brachte in ähnlicher Weise wie bei der gesunden Niere, die Oligurie hervor, deren Grad aber stärker als bei der gesunden Niere war. Im III. Stadium wurde jedoch die Harnbildung wiederhergestellt. Die Abnahme der Zufuhrmenge zur Nierenarterie erfolgte in gleichem Masse wie bei der gesunden Niere. Ein Beispiel dafür ist mit Fig. 4. illustriert.

b) Zufuhr von der Nierenpfortader aus.

Einer von 5 Versuchen ist mit Fig. 5. illustriert.

Im II. Stadium verhielt sich die Harnabsonderung ausnahmslos oligurisch, im III. Stadium trat die Wiederstellung der Harnbildung ein. Der Grad der Oligurie war gewissermassen stärker als bei der gesunden Niere, die Wiederherstellung der
Fig. 4. Kantharidinniere; Durchspülnng mit $1 \% 0$ iger Gummi-Ringerlösung durch Nierenarterie.

Kröte, 今, Körpergewicht $150 \mathrm{~g}$. Gummi-Ringer 。

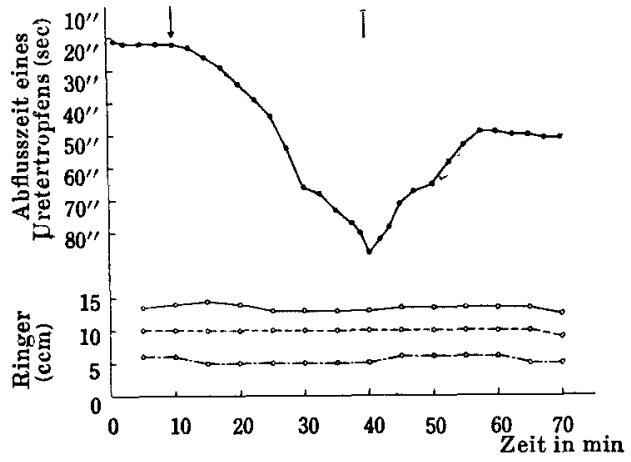

Ausfuhrmenge von V. cava post.

$\ldots$ Zufuhrmenge in Nierenpfortader

Zufuhrmenge in Nierenarterie

Fig. 5. Kantharidinniere; Durchspülung mit $1 \%$ iger Gummi-Ringerlösung durch Nierenptortader.

Kröte, \&, Körpergewicht $180 \mathrm{~g}$. Gummi-Ringer

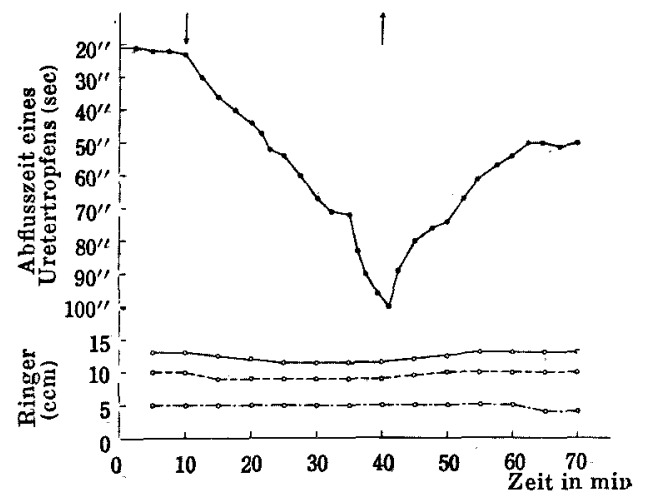
\begin{tabular}{l} 
Ausfuhrmenge von V. cava post. \\
\hdashline- Zufuhrmenge in Nierenpfortader $^{-}$
\end{tabular} 
Harnbildung im III. Stadium erfolgte etwas langsamer. Die $\mathbf{A b}$ nahme der Zufuhrmenge zur Nierenpfortader vollzog sich in annähernd gleichem Masse wie bei der gesunden Niere.

c) Gleichzeitige ZufuhrvonderNierenarterieund von

Fig. 6. Kantharidinniere; Durehspülung mit $1 \%$ iger Gummi-Ringerlösung durch Nierenarterie und Nierenpfortader.

Kröte, $\delta$, Körpergewicht $130 \mathrm{~g}$. Gummi-Ringer

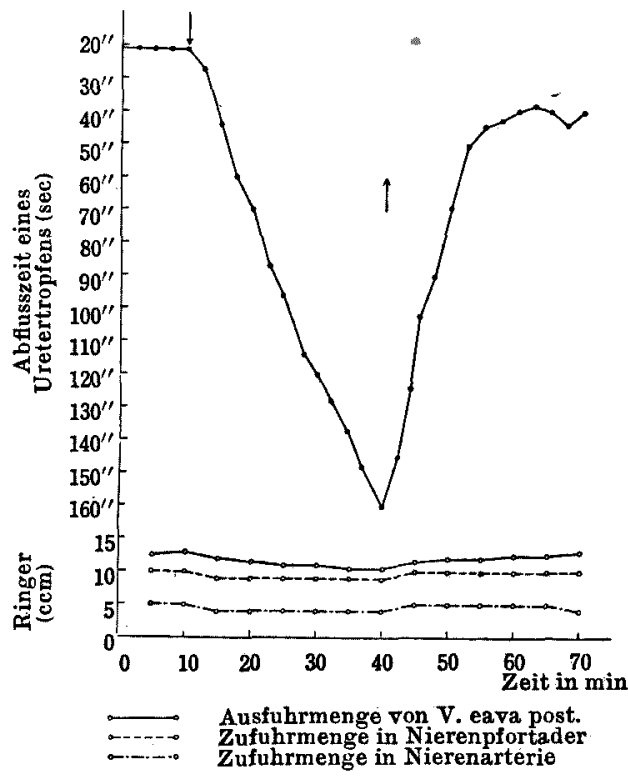

3. Versuch'an der Uranniere.

a) Zufuhr von der Nierenarterie aus.

Einer von 5 Versuchen ist als ein Beispiel dafür mit Fig. 7. illustriert.

Die Bildung des Uretertropfens im I. Stadium zeigte keinen besonderen Unterschied von derselben bei gesunden Niere, im II. Stadium aber trat ein bemerkenswerter Unterschied auf. Es wurde nämlich diejenige Oligurie zutage der Nie-ren pfortaderaus.

An 5 Versuchen wurde die Harnbildung untersucht. (Fig. 6).

Im I. Stadium wurde jedwede Abweichung von der Norm vermisst, der Grad der Oligurie im II. Stadium war stärker und die Wiederherstellung der Harnbildung im III. Stadium erfolgte ebenso deutlith wie bei der gesunden Niere. Die Abnahme der Zufuhrmenge zeigte sich kaum verschieden von der bei der gesunden Niere.

Fig. 7. Uranniere; Durchspülnng mit $1 \%$ iger Gummi-Ringerlösung. durch Nierenarterie.

Kröte, 우, Körpergewicht $239 \mathrm{~g}$. Gummi-Ringer

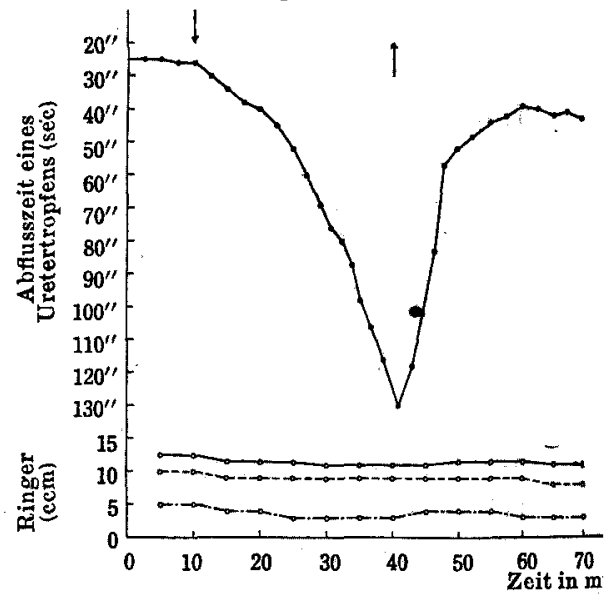

- Ausfuhrmenge von V. cara post. Zufuhrmenge in Nierenpfortader Zufuhrmenge in Nierenarterie 
gefördert, welche weitgehend intensiver war sowohl als bei der gesunden wie auch als bei der Kantharidinniere. Im U. Stadium wurde dennoch das Verhalten des Uretertropfens ebenso prompt wie bei der gesunden Niere wiederhergestellt. Die Abnahme der glomerulären Zufuhrmenge im III. Stadium erfolgte mit dem Zeitablauf in weiter gewissermassen stärkerem Masse als bei den gesunden Niere, und zudem ging die Wiederherstellung im III. Stadium nicht so vollkommen wie im I. Stadium vonstatten, wie dies bei der gesunden Niere der Fall war. Hierbei gab es 3 Versuche, in denen im II. Stadium die Zufuhrmenge auch im Pfortadersystem mehr oder minder abnahm, in anderen 2 Versuchen wurde derartige Abnahme kaum angetroffen. Im III. Stadium neigte die Zufuhrmenge zu mehr oder weniger Abnahme.

b) Zufuhr von der Nierenpfortader aus.

An 5 Versuchen wurde das Experiment angestellt.

Im II. Stadium trat auffallende Oligurie ohne Ausnahme auf, das Intervall des Austropfens des Uretertropfens erstreckte sich auf 5 Min. 20 Sek. bzw. auf 7 Min. 25 Sek. Die Abnahme der Zufuhrmenge zur Nierenpfortader, welche beim Übergang in das II. Stadium auftrat, war etwas stärker als bei der gesunden Niere, und die Wiederherstellung im III. Stadium vollzog sich nicht allein nicht bis zu gleichem Niveau im I. Stadium, wie bei der gesunden Niere, sondern einer geringfügige Gefässwirkung bestand noch weiterfort, nachdem die Harnbildung sich wiederherzustellen angefangen hatte. Die Zufuhrmenge zur Nierenarterie im II. Stadium liess in 2 Versuchen keine Veränderung erkennen, während

Fig. 8. Uranniere; Durchspülung mit 1\% iger Gummi-Ringerlösung durch Nierenpfortader.

Kröte, 官, Körpergewicht $160 \mathrm{~g}$. Gummi-Ringer

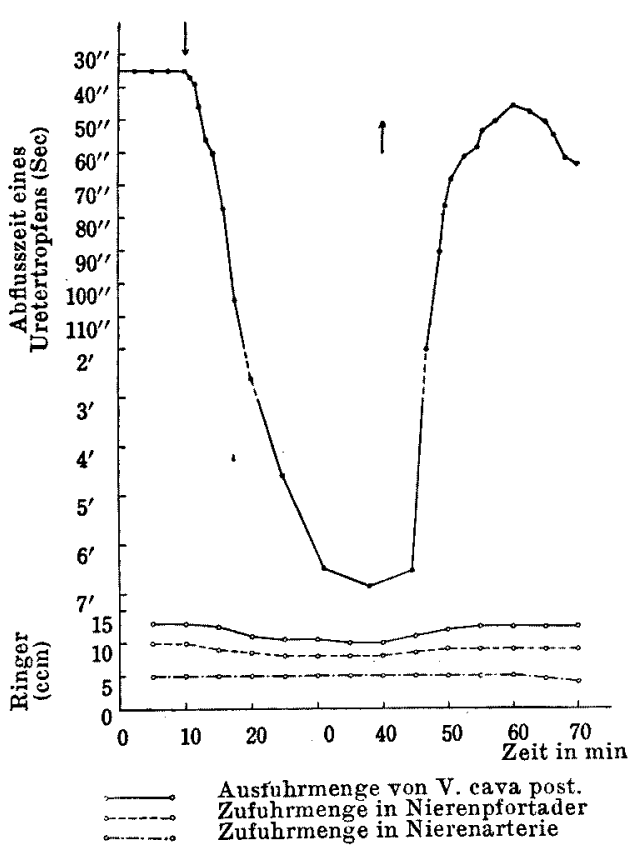
sie in übrigen 3 Versuchen äusserst spärlich abnahm. Im III. Stadium 
tendierte diese Zufuhrmenge mit dem Zeitablauf zu mehr oder minder Abnahme. Fig. 8. veranschaulicht diese Verhältinisse bei einem Versuch.

c) Gleichzeitige Zufuhr von der Nierenarterie und von der Nierenpfortader aus.

Einer von 5 Versuchen ist mit Fig. 9 illustriert.

Geradezu eklatant war die Oligurie und zwar derart, dass sie sich in 2 von 5 Versuchen bis zum Zustand der Anurie ausartete, gleichwohl

Fig. 9. Uranniere; Durchspülung mit 1\% iger Gummi-Ringerlösung durch Nierenarterie u. Nierenpfortader.

Kröte, 우, Körpergewicht $287 \mathrm{~g}$.

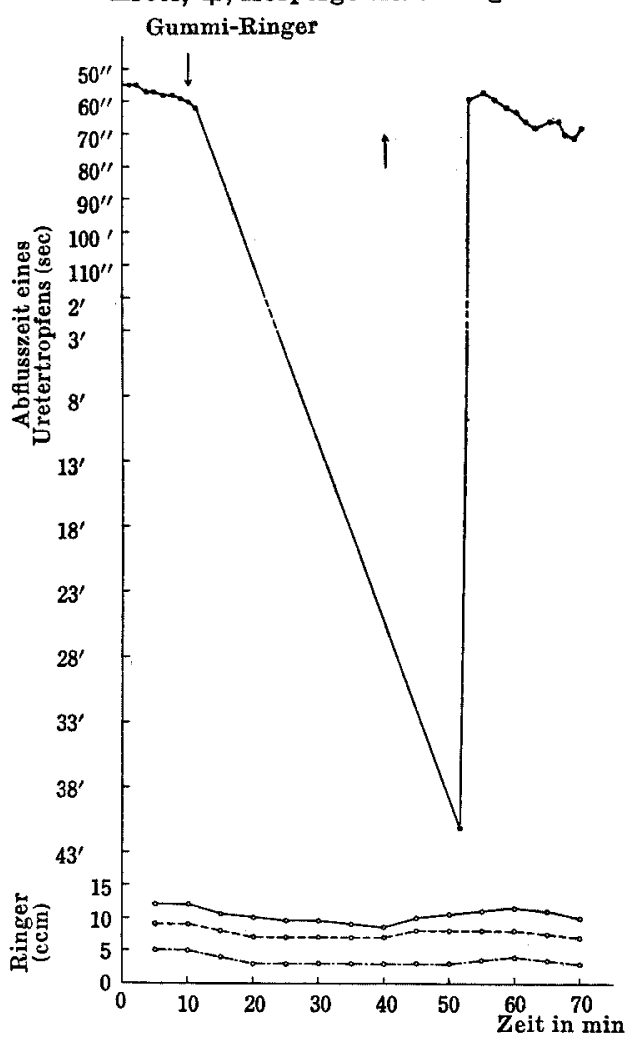

Ausfuhrmenge von V. cava post. a...... Zufuhrmenge in Nierenarterie trat im III. Stadium die Wiederherstellung sehr rasch ein.

Das Intervall des Austropfens des Uretertropfens erreichte ja 31 Min. 15 Sek. bzw. 41 Min. 6 Sek. Mit anderen Worten, es bildete sich in oben erwähnter Zeitspanne nur ein Tropfen Harn.

Die Abnahme der Zufuhrmenge war ziemlich grösser als bei der gesunden Niere, in 2 Versuchen bestand eine geringtügige $\mathrm{Ge}$ fässwirkung an der Nierenarterie auch nach dem Übergang in das III. Stadium noch fort. Die Zufuhrmenge im III. Stadium kam dann nicht bis auf den vollauf gleichgrossen Wert wie der im 1. Stadium zurück, nachdem die Harnbildung sich schon wiederhergestellt Nach alledem ist anzunehmen, dass hier die Gefässwirkung noch fortbestand.

Oben ist der Einfluss 1\% iger Gummi-Ringerlösung auf die in- 
trarenale Harnbildung unter verschiedenen Massnahmen, indem man nämlich isolierte äberlebende Krötenniere mit obiger Lösung entweder von dem glomerulären oder von dem renoportalen Kreislauf aus getrennt oder von den beiden Systemen aus gleichzeitig durchströmte, beobechtet worden; es zeigte sich, dass es bei jeder Art der Durchspülung unter gleichem Verlauf zur Oligurie kommt. Und sofort nach Umschaltung auf die Normal-Ringerlösung tritt die Erholung von der Oligurie ein.

Es hat sich weiterhin herausgestellt, dass je nach Art der Versuchsanordnungen und je nach Graden der Nierenschädigungen auch die Oligurie in verschieden starkem Grad auftritt. Bei glomerulärer Durchspülung nämlich tritt die Oligurie zwar deutlich, doch höchst langsamer als bei allen anderen Durchspülungsarten ein, bei tubulärer Durchspülung ist sie etwas stärker und bei gleichzeitig ausgeführter glomerulärer und tubulärer Durchspülung tritt sie aufs deutlichste in Erscheinung.

Auch bei Kantharidinniere ist ein annähernd gleiches Verhalten anzutreffen und zwar tritt die Oligurie in allen Versuchen in stärkerem Grad als bei der gesunden Niere hervor. In bezug auf die Zufuhmenge ist kaum jedweder Unterschied zwischen der Kantharidinniere und der gesunden Niere nachzuweisen. Bei Uranniere tritt die Oligurie im II. Stadium derart imponierend in den Vordergrund, dass sie dieselbe sowohl bei der gesunden wie auch bei Kantharidinniere an Intensität weitaus übertrifft. Im übrigen ist zu bemerken, dass die Oligurie hier bei renoportaler Durchspülung stärker als bei glomerulärer Durchspülung ist und bei gleichzeitiger Durchspülung von den beiden System aus sich soweit progressiv kundgibt, dass man diese Oligurie nicht mit Unrecht eher als Anurie ansprechen möchte. Die derart arg betroffene Harnbildung schlägt dennoch sogleich nach Umschaltung auf die Normal-Ringerlösung in die prompte und lebhafte Harnbildung um. Der vollzogenen Harnbildung entsprechend tritt auch die Veränderung in der Zufuhrmenge der Spülflüssigkeit auf, deren Grad aber nicht so bemerkenswert ist. Alles in allem lässt sich also sagen, dass diese ausgeprägte Veränderung der Harnbildung sich unmöglieh durch die Gefässwirkung allein erklären lässt.

Nun sei im folgenden darüber betrachtet, durch was für einen Mechanismus oben auseinandergesetzte Versuchsergebnisse hervorgebracht worden sind.

Es liegt zunächst auf der Hand,dass bei glomerulärer Durchspülung die durch Anwesenheit des in der Spülflüssigkeit enthaltenen 
Gummi-Arabicums bedingte Steigerung kolloid-osmotischen Drucks in den Glomerulusschlingen zur Herabminderung der Filtration und infolgedessen auch zur Oligurie führt. Hierbei muss aber auch die Abnahme der Zufuhrmenge berücksichtigt werden.

Vorderhand muss dahin gestellt bleiben die Frage danach, durch welche Ursache die im II. Stadium aufgetretene, die Gefässwirkung vertäuschende Abnahme der Zufuhrmenge ausgelöst würde.

Sato ${ }^{11}$ konnte im Durchspülungsversuch mit $2 \%$ iger GummiRingerlösung am Schenkelpräparat des Kaninchens eine geringfügige vasokonstriktorische Wirkung als Ausdruck der Gefässwirkung des Gummi-Arabciums nachweisen. Tanno ${ }^{12)}$ hat im Durchspülungsversuch am Mesenterium des Frosches festgestellt, dass sofort nach Umschaltung von Ringerlösung auf Gummi-Ringer die Abnahme der Tropfenzahl und die Verminderung der Spülflüssigkeitsmenge auf: treten, während bei erneuter Umschaltung auf Normal-Ringer sofortige Wiederherstellung der Tropfenzahl und die Zunahme der Zuführmenge zutage treten. Auch Inoue ${ }^{13)}$ hat im Durchspïlnngsversuch an der Krötenniere unter Anwendung der Gummi-Ringerlösung von verschieden starken Konzentrationen die Abnahme der Zufuhrmenge nachgewiesen.

Die Gummilösung reagiert eigentlich schwach sauer; weil aber die in vorliegenden Versuchen verwendete Gummilösung zuvor mit dem Natrium karbonat aufs strengste neutralisiert worden war, haben wir keinen Grund, anzunehmen, dass die hier in Betracht kommende Gefässwirkung durch Erhöhung der Wasserstoffionenkonzentration der Spülflüssigkeit herbeigeführt worden wäre. Andererseits kann man aber auch mit der Möglichkeit rechnen, dass es wegen hochgradiger Viskosität der Gummi-Ringerlösung zur Verlangsamung der Durchspülung, folglich zur Abnahme der Zufuhrmenge kommen könnte. Wie dem auch sei, ist es-angesichts der Tatsache, dass, trotzdem die Oligurie sich mit dem Zeitablauf in immer stärkerem Grad kundgibt, die Abnahme der Zufuhrmenge keineswegs mit dieser Verstärkung der Oligurie gleichen Schritt hält-durchaus nicht angängig, diese Abnahme der Zufuhrmenge allein als die Hauptursache für die Oligurie hinzustellen. Nun sei wieder auf unsere Ergebnisse eingegangen.

Wenn die Spülflüssigkeit von Gummi-Ringer auf Normal-Ringer

11) S a to, Tohokn Journ. Exp. Med., 1928, 9, 489.

12) Tann o, Seiikai Zasshi, 1936, 55, 1168.

13) In o ue, Ibid., 1937, 56, 1888. 
umgeschalt worden ist, wird die Oligurie zwar rasch wiederhergestellt, nicht aber bis zu gleichem Niveau wie im 1. Stadium. Utberdies ist das Intervall des austropfenden Uretertropfens, welches sich nach oben angeführter Umschaltung erholte und eine Zeitlang konstant geblieben war, nachher wieder zur Verlangsamung geneigt. Diese Verlangsamung spricht offenbar dafür, dass das betreffende Präparat auch selbst bei Durchspülung mit Normal-Ringer infolge allmählicher Abschwächung durch $\mathrm{O}_{2}$-Mangel schliesslich der Oligurie anheimfällt. Die Ursache dafür, dass bei renopfortaler Durchspülung mit GummiRinger, trotz fehlender Veränderung der Filtration in Glomerulusschlingen, erhebliche Oligurie zutage tritt, muss sicherlich daringelegen sein, dass die wasseranziehende Kraft, welche der im das Tubuluslumen umschlingenden Pfortader-Kapillarsystem befindlichen Spülungsflüssigkeit zukommt, also der kolloidosmotische Druck den im Tubuluslumen vorhandenen Glomerulusharn stark zu sich aufsaugt, wobei die Nierenepithelien durch Wirkung der Gummilösung stark geschädigt und in ihrer Funktion beeinträchtigt sind, infolgedessen ihre beschränkende bzw. selektive Kraft auf die Rückresorption herabgesetzt ist, wodurch die Rïckresorption sich abnorm steigert. Dass durch starke und rasche Veränderungen des osmotischen Drucks des Blutes bzw. der Gewebsflüssigkeit Veränderungen in der Vitalität des Organismus zustande kommen, ist durch Untersuchungen von $\mathrm{Falk}^{\mathbf{1 4})}$, Bosc u. Vedel ${ }^{15)}$, Heilner ${ }^{16)}$, Rolly u. Christjansen ${ }^{17)}$, Prig$\mathbf{g e}^{18)}$, Underhill u. Sallik ${ }^{19)}$ u. a. erwiesen worden. Mit Rücksicht auf die Zufuhrmenge ist an der Nierenarterie keine besondere Gefässwirkung nachweisbar. Bei Durchspülung mit Gummi-Ringer von den renoportalen Kreislauf aus ist eine äusserst winzige Abnahme der Zufuhrmenge anzutreffen. Diese Abnahme hätte theoretisch eine Herabsetzung der Rückresorption in den Tubulusepithelien nach sich ziehen sollen, in Wirklichkeit aber ist die Rückresorption hierbei zu gross, um durch derartige geringfügige Abminderung der Zufuhrmenge beeinflusst zu werden.

Es liegt selbstverständlicherweise auf der Hand, dass, weil bei gleichzeitiger Durchspülung von glomerulären sowie tubulären Kreis-

14) Falk, Arch. f. path. Anat. u. Physiol, 1872, 56, 315 .

15) Bosc a. Vedel, C. r. Soc. Biol., 1896, 48, 612, 736 u. 749 ; Arch. Physiol. norm. et pathol., $1896,8,937$.

16) Heilner; Zeitschr. f. Biol., 1907, 49, 373.

17) Rolly u. Christjansen, Arch. f. exp. Pathol. u. Pharm., 1914, 77, 34.

18) Prigge, Deutsch. Arch. klin. Med., 1922, 139, 1.

19) Underhill u. Sollik, Journ. Biol. Chem., 1925, 63, 61. 
lauf aus eine beträchtliche Abnahme der Filtration in den Glomertlusschlingen, wie bereits oben angeführt, stattfindet, und dazu noch eine abnorme Steigerung der Rückresorption in den Tubuli hinzutritt; durch den Synergismus der beiden Wirkungen die Oligurie ausserordentlich intensiv zutage gefördert wird.

Die Abnahme der Zufuhrmenge als Gefässwirkung ist hierbei sowohl an der Nierenarterie wie auch an der Nierenpfortader anzutreffen; und der Verlauf der beiden Abnahmen geht beinahe zueinander parallel. Es kann zwar nicht geleugnet werden, dass die Abnahme der Zufuhrmenge zur Nierenarterie einen Faktor für die Oligurie darstellt; die Abnahme der Zufuhrmenge zur Nierenpfortader wirkt aber, wie oben erwähnt, der Oligurie entgegen. Und wenn die Oligurie schon mit dem Zeitablauf immer mehr an Intensität zunimmt, hält die Abnahme der Zufuhrmenge doch nicht immer damit gleichen Schritt, demnach ist eine derartige Gefässwirkung durchaus nicht als ein bedeutsamer Faktor für die Oligurie anzusprechen.

Bei der Kantharidinniere tritt bei glomerulärer Durchspülung mit Gummi-Ringer die Oligurie in stärkerem Masse als bei der gesunden Niere auf. Bei der Kantharidinniere, besonders bei glomerulärer Durchspülung mit Gummi-Ringer ist die Filtration durch geschädigte Glomerulusschlingen deshalb um so stärker herabgesetzt, weil die Flüssigkeit mit dem hohen kolloid-osmotischen Druck in den Endothelschlingen vorhanden ist, und zweitens, weil hierbei nicht allein die Glomeruli, sondern die Tubulusepithelien auch mehr oder weniger in Mitleidenschaft gezogen werden müssen, folglich die funktionelle Beeinträchtigung der Nierenepithelien besteht, tritt die regulierende bzw. beschränkende Wirkung der Harnkanälchenepithelien auf die Rückresorption in den Hintergrund, nun erst recht gewinnt die Rückresorption des Wassers die Oberhand, daraus folgt zwangsläufig die Abnahme der Harnmenge.

Es haben Yamaguchi u. seine Mitarbeiter ${ }^{20)}$ das Kongorot, Adachi u. Kasugai ${ }^{21)}$ den Harnstoff, Shoji u. Takeda ${ }^{22}$ das Kreatinin von den Ureteren aus direkt in das Tubuluslumen einfliessen lassen, Take da $\mathrm{a}^{23)}$ hat den Durchspülungsversuch mit Trypanblau vorgenommen; alle genannte Forscher haben den an normalen Nierenepithelien niemals beobachtbaren Durchgang der oben erwähnten Sub-

20) Yamaguchi, Takahashi, Takeda u. Shoji, Tohoku Journ. Exp. Med., $1933,20,331$.

21) Ad acti u. Kasugai, Ibid., 1933, 20, 350.

22) Taked a u. Sho ji, Ibid., 1935, 26, 592.

23) Taked a, Ibid., 1935, 27, 38. 
stanzen an den Nierenepithelien sowohl der Kantharidinniere wie auch der Uranniere deutlich wahrgenommen und dadurch den Beweis dafür erbracht, dass die Giftwirkung des Kantharidins sich nicht allein streng auf das Gebiet der Glomeruli lokalisiert, sondern bis zu einem gewissen Grad auch weiterhin auf die Nierenepithelien übergreifen kann.

Auch selbst bei renoportaler Durchspülung mit Gummi-Ringer erfolgt die Oligurie in stärkerem Masse als bei der gesunden Niere. Dies erklärt sich folgenderweise: Weil die kolloidalen Lösung mit hohen osmotischen Druck nämlich auf die Nierenepithelien, welche nicht so grob anatomisch, so doch wenigstens funktionell schon gestört sind, von ihren Rückenseite, d.h. von der Gefässseite her einwirkt, ist die Nierenepithelfunktion, die Rückresorption einzuschränken, herabgesetzt, und durch den Hinzutritt des wasseranziehenden osmotischen Wirkung von ihrer Rückenseite her wird diese Funktionsstörung der Nierenepithelien verdoppelt, so dass die Rückresorption erheblich zuwächst, was zwangsläufig zu weiterer Verstärkung der Oligurie führen muss. Aus dem Umstand, dass trotzdem beim Ausbleiben der Abnahme der Zufuhrmenge zur Nierenarterie an der mit der GummiRingerlösung durchströmten Nierenpfortader eine geringfügige Abnahme der Zufuhrmenge erkennbar ist, eine derart starke Oligurie zutage gefördert wird, kann man wohl ersehen, wie gross die wasseranziehende Kraft der kolloidalen Lösung ist.

Der Grund dafür, dass die Oligurie, welche bei der Kantharidinniere bei gleichzeitiger Durchspülung mit Gummi-Ringer von der Nierenarterie und Nierenpfortader aus in Erscheinung tritt, stärker ist als alle anderweitige Oligurien, muss begreiflicherweise im Zusammentreffen der herabgesetzten Glomerulusfiltration und der gesteigerten Rückresorption gelegen sein.

Da bei der Uranniere vornehmlich die Tubuli aufs stärkste geschädigt sind, und deshalb die Steigerung der Rückresorption aufs intensivste sich vollzieht, kommt es zu weitgehend stärkerer Oligurie als bei der gesunden sowie Kantharidinniere. Da bei glomerulärer Durchströmung mit Gummi-Ringer durch Abnahme der Wasserfiltration schon der Glomerulusharn abgemindert ist, zumal durch Funktionsstörung derTubulusepithelien eine abnorm gesteigerte Rückresorption statthat, kommt es zu um so stärkerer Verminderung der Harnmenge. Auch in diesem Fall hat man keinen Grund, die allmähliche Abnahme der Zufuhrmenge zur Nierenarterie als die Hauptursache für die Oligurie anzunehmen, weil diese Abnahme noch fortbesteht, nachdem 
nach vollzogenem Umschalten auf die Normal-Ringerlösung die Harnbildung schon lebhaft vor sich zu gehen angefangen hat.

Beim Einwirkenlassen der Gummi-Ringerlösung von dem renoportalen Kreislauf aus müssen die bereits in ihrer Funktion gestörten Tublusepithelien durch die wasseranziehende Kraft der im Pfortaderkapillarsystem vorhandenen Spülflüssigkeit selbst noch erneut stark beeinflusst werden, es tritt dann die Oligurie um so stärker hervor, als auf solche Weise der Rückresorption Vorschub geleistet wird. Was die Zufuhrmenge hierbei anbelangt, ist sie in der Nierenpfortader im Vergleich mit der gesunden Niere einigermassen herabgesetzt. Diese Herabsetzung bezieht sich auf die Gefässkontraktion, welche sich als Ausdruck der Reaktion der Nierenpfortader auf die Spiulfllüssigkeit kundgibt. Und der Umstand, dass hierbei manchmal auch an der Nierenarterie, trotz der Durchspülung mit Normal-Ringer, eine Gefässwirkung sich äussert, dürfte höchstwahrscheinlich auf die Beeinflussung der Vasa afferentia durch die Gummilösung welche vermittelst. der zwischen dem Nierenarterien und Pfortader-System bestehenden Kommunikation zur Einwirkung gelangt, zurïckzuführen sein.

Zum Schluss ist noch hervorzuheben, dass bei gleichzeitiger Durchspülung von dem glomerulären sowie von dem renoportalen Kreislauf aus die Harnbildung durch kombinierte Einflüsse der von dem glomerulären sowie von dem rénoportalen Kreislauf aus gesondert zur Einwirkung gelangenden Gummilösungen in um so stärkerem Masse Verminderungen erfährt. Es ist also nicht zu verwundern, dass es bei starkgradigen Schädigungen der Tubulusepithelien zur Anurie kommt. Der Umstand, dass diese Anurie durch Durchspülung mit Normal-Ringer rasch zurücktritt, und im Anschluss daran die Harnbildung lebhaft stattzuhaben anfängt, ist als ein Hinweis auf eklatante Wirkung kolloidaler Lösungen auf die Harnbildung von grossem Interesse.

\section{Zusammenfassung.}

Es wurden isolierte überlebende Krötennieren im gesunden Zustand sowie nach Vergiftung mit Kantharidin sowie Uran mit 1\%iger Gummi-Ringerlösung durchspült.

1. Bei glomeruläre Durchspülung erfährt die Harnabsonderung ausnahmslos bedeutende Verminderung. Beim Umschalten der Spülflüssigkeit auf Normal-Ringer ist diese Oligurie rasch und deutlich wiederhergestellt. Die durch Durchspülung mit Gummi-Ringer herbeigeführte Oligurie ist bei der Uranniere am stärksten, bei der Kan- 
tharidinniere weniger stark und bei der gesunden Niere zwar erheblich, aber immerhin weniger intensiv als bei den 2 erstgenannten Nieren. Für derartige Oligurie dürfte die Gefässwirkung an der A. renalis auch ein Moment abgeben, diese ist aber verhältnismässig geringfügig. Diese Oligurie muss meines Erachtens dadurch ausgelöst werden, dass die Filtration in den Glomerulusschlingen durch das Vorhandensein eines Kolloides, des Gummi arabicums, d.h. durch Steigerung des der Spülflüssigkeit zukommenden kolloid-osmotischen Drucks gehemmt wird. Die hierbei entstandene Gefässwirkung dürfte wahrscheinlich durch eine durch gesteigerte Viskosität der Gummi-Ringerlösung verursachte Erhöhung der Reibung derselben und der Gefässwand ausgelöst worden sein.

2. Bei renoportaler.Durchspülung tritt Oligurie bei allen Nierenarten deutlich auf; diese Oligurie ist bei der Uranniere höchstgradig, bei der Kantharidinniere hochgradig; bei der gesunden Niere geringgradig: Allerdings sind die Grade der Oligurie im allgemeinen hier einigermassen stärker als bei glomerulärer Durchspülung. Nach dem Umschalten auf die Normal-Ringerlösung schlägt diese Oligurie in lebhafte Harnbildung um. Diese intensive Oligurie beruht auf abnormer Steigerung der Rückresorption, welche durch Herabsetzung der hemmenden Wiekung auf die Rückresorption herbeigeführt worden ist.

3. Bei gleichzeitiger Durchspülung von dem glomerulären und tubulären Kreislauf aus kommt die Oligurie im stärkeren Grad zustande, als sie bei entweder von dem glomerulären oder tubulären Kreislauf aus getrennt vorgenommener Durchspülung, wie oben erwähnt, in Erscheinung tritt. Und diese Oligurie kommt bei der Uranniere aufs intensivste zum Vorschein und zwar derart, dass sie sich bis zum Zustand der Anurie steigert. Auch in diesem Fall, sofort nach dem Umschalten der Spülflüssigkeit auf Normal-Ringer tritt bemerkbare Wiederherstellung der Harnmenge ein.

Aus oben angeführten Ergebnissen geht klar hervor, dass durch Kolloidzusatz zur Nährflüssigkeit die Glomerulusfiltration stark gehemmt ist, wobei auch die Funktion der Nierenepithelien herabgesetzt ist und dass es zu abnormer Steigerung der Wasserrückresorption, folglich zur Oligurie kommt.

Für die wertvolle Unterstützung bei vorliegender Arbeit spreche ich dem seligen Herrn Dr. Yamaguchi, Dozenten an hiesiger Klinik, meinen herzlichen Dank aus. 